\title{
Oxigenoterapia inalatória em pacientes pediátricos internados em hospital universitário
}

\author{
Oxygen inhalation therapy in children admitted to an university hospital
}

\author{
Paula Angeleli B. de Camargo ${ }^{1}$, Amanda Tavares Pinheiro ${ }^{1}$, Ana Carolina R. Hercos ${ }^{1}$, Gisela Fleischer Ferrari²
}

\section{RESUMO}

Objetivo: Avaliar o uso da oxigenoterapia inalatória em crianças internadas em hospital universitário.

Metódos: Estudo prospectivo de crianças atendidas no Pronto-Socorro Pediátrico do Hospital das Clínicas da Faculdade de Medicina de Botucatu e que receberam oxigenoterapia durante a internação, de maio a setembro de 2005. Indicou-se oxigenoterapia se saturação de oxigênio inferior a $90 \%$ e frequência respiratória elevada para idade. Crianças em uso crônico de oxigênio ou com necessidade de ventilação mecânica foram excluídas. Foram avaliados: sintomas respiratórios, diagnósticos clínicos, saturação de oxigênio, método e tempo de oxigenoterapia e responsável pela prescrição.

Resultados: Foram atendidas 8.709 crianças no prontosocorro, sendo que 2.769 (32\%) apresentaram doenças respiratórias e 97 necessitaram de internação na enfermaria. Destas, $62(64 \%)$ receberam oxigenoterapia. Das 62 crianças, 37 eram do sexo masculino e a idade variou de 2 meses a 14 anos (mediana: 8 meses). A causa de hipóxia foi pneumonia em 52 crianças (84\%), asma em cinco, bronquiolite em quatro e traqueomalácia em uma. As prescrições de oxigenoterapia foram feitas por médicos, com monitoração de saturação de oxigênio por oxímetro de pulso. O tempo mediano de administração de $\mathrm{O}_{2}$ foi 6 dias e o cateter nasal foi usado em $94 \%$ dos casos, sendo raro o uso de máscaras ou capuz de oxigênio.

Conclusões: A oxigenoterapia inalatória foi mais frequente em crianças com menor idade e em pacientes com pneumonia, sendo sua indicação compatível com critérios internacionais. $\mathrm{O}$ uso do cateter nasal mostrou-se seguro, simples, efetivo e de baixo custo.

Palavras-chave: oxigênio; oxigenoterapia; criança; unidades hospitalares.

\section{ABSTRACT}

Objective: To evaluate inalatory oxygen therapy in children admitted to a university hospital.

Methods: Prospective study of children assisted at the Emergency Room of the University Hospital of Botucatu Medical School and submitted to oxygen therapy during hospitalization, from May to September 2005. Criteria for oxygen therapy were oxygen saturation less than $90 \%$ and high respiratory rate for age. Children in chronic use of oxygen or in need of mechanical ventilation were excluded. The following data were analysed: clinical respiratory symptoms, clinical diagnosis, oxygen saturation, methods, duration and who made the prescription of oxygen therapy.

Results: Out of 8,709 children admitted to the emergency room, 2,769 (32\%) had respiratory tract diseases and 97 needed hospitalization. From these, 62 (64\%) were submitted to oxygen therapy ( 37 males; 2 months to 14 years old). Pneumonia was the cause of hypoxemia in 52 children (84\%), asthma in five, bronchiolitis in four and tracheomalacia in one. Oxygen therapy was prescribed by physicians, with daily observation and monitoring of oxygen saturation by pulse oxymetry. The median time of oxigen use was 6 days and nasal catheters were used in $94 \%$ of children, Facial masks or hoods were rarely prescribed.

Conclusions: Inalatory oxygen therapy was more frequently used in younger patients and in children with pneumonia. Its prescriptions followed international criteria. Nasal catheter seemed to be safe, simple, effective and an inexpensive method of oxygen delivery.

Key-words: oxygen; oxygen inhalation therapy; child; hospital units.

Caixa Postal 530 - Distrito de Rubião Júnior

CEP 18618-970 - Botucatu/SP

E-mail: paula.angeleli@gmail.com

Fonte financiadora do projeto: Fundação de Amparo à Pesquisa do Estado de São Paulo (Fapesp), processo 06/54871-9.

Recebido em: 6/9/2007

Aprovado em: 7/1/2007 


\section{Introdução}

O oxigênio teve seu caráter vital reconhecido desde a sua descoberta, realizada de forma independente por Schelee, em 1772, e por Pristly, em 1774. Já em 1780, foi utilizado com propósitos médicos por Chaussier, que empregou o oxigênio em recém-nascidos com dificuldade respiratória ${ }^{(1)}$. Desde então, a administração de oxigênio vem sendo uma das mais importantes modalidades de terapia para pacientes com hipóxia resultante de condições comuns como infecções do trato respiratório inferior que, quando graves, podem levar à morte ou a seqüelas em qualquer idade ${ }^{(2,3)}$. Além dessa indicação, o gás também tem seu valor terapêutico comprovado em outras situações, como a diminuição da incidência e gravidade da apnéia em prematuros e a dilatação das artérias pulmonares com conseqüente aumento do fluxo sanguíneo pulmonar ${ }^{(4)}$.

As infecções do trato respiratório inferior - particularmente pneumonias - são a principal causa de morte em crianças menores de cinco anos de idade nos países em desenvolvimento ${ }^{(5)}$, sendo que, no mundo, são responsáveis pela morte anual de mais de 4 milhões de crianças nessa faixa etária ${ }^{(6,7)}$. No Brasil, em 2002, tais doenças foram responsáveis por 3.725 óbitos (5\%) de um total de 69.093 mortes em crianças menores de cinco anos ${ }^{(8)}$. Nos pacientes hipoxêmicos, o emprego do oxigênio é responsável por uma substancial redução no número de mortes ${ }^{(6)}$.

A oxigenoterapia consiste no tratamento da hipóxia por meio da inalação de oxigênio, a uma pressão maior que a do ar ambiente, o que facilita a troca gasosa e reduz o trabalho da respiração $0^{(9)}$. O oxigênio usado deve ser umidificado e aquecido. A escolha da forma de administração dependerá, principalmente, da eficiência do sistema a ser empregado ${ }^{(10)}$. Cateteres nasais, de fácil instalação, proporcionam uma fração inspirada de oxigênio $\left(\mathrm{FiO}_{2}\right)$ entre 24 e $40 \%$, porém, se deslocam facilmente. $\mathrm{O}$ uso de máscaras nasais também é simples e fornece $\mathrm{FiO}_{2}$ de até $60 \%$; contudo, apresenta desvantagens como difícil fixação, interferência na alimentação, expectoração e aspiração de vias aéreas. $\mathrm{O}$ capuz para recém-nascidos e a oxitenda para lactentes e pré-escolares proporcionam, respectivamente, $\mathrm{FiO}_{2}$ de até 100 e $60 \%$. Ambos devem usar gás aquecido e têm, como maiores problemas, o ruído em seu interior e a dificuldade com a alimentação e com a aspiração de vias aéreas ${ }^{(11)}$, além do isolamento em relação ao ambiente imposto sobre a criança ${ }^{(12)}$.

Mesmo sendo essencial à vida, o oxigênio, como qualquer medicamento, quando administrado de forma indevida, pode ser tóxico e ter sérias consequiências. A toxicidade, que depende de fatores como pressão absoluta de oxigênio oferecido, duração da exposição e sensibilidade individual, é a maior limitação é à sua administração( ${ }^{(4,13,14)}$.

Dentre os efeitos colaterais do uso de oxigênio, observamse: retinopatia da prematuridade, doença pulmonar crônica e displasia broncopulmonar em prematuros, atelectasia por altas concentrações de oxigênio e dano epitelial pulmonar devido ao estresse oxidativo ${ }^{(4)}$. A toxicidade do oxigênio pode causar traqueobronquite, depressão da atividade mucociliar, náuseas, anorexia e cefaléia - situações reversíveis com a suspensão da oxigenoterapia. Por outro lado, a saturação muito baixa de oxigênio pode resultar em aumento da resistência pulmonar, limitação do crescimento somático e morte súbita em crianças com doença pulmonar crônica ${ }^{(10)}$. Assim, recomenda-se a oxigenoterapia após avaliação rigorosa quanto à real necessidade de sua utilização e, durante seu uso, monitoração contínua de todos os parâmetros do paciente.

O presente estudo teve como objetivo avaliar os critérios utilizados para indicar e interromper a oxigenoterapia, a forma de administração do oxigênio, a monitoração do paciente em oxigenoterapia e as doenças envolvidas para a indicação desta terapia nos pacientes da Enfermaria de Pediatria do Hospital das Clínicas da Faculdade de Medicina de Botucatu da Universidade Estadual Paulista (Unesp).

\section{Métodos}

Trata-se de estudo prospectivo, descritivo, que avaliou todos os pacientes atendidos por doenças respiratórias no ProntoSocorro Pediátrico do Hospital das Clínicas da Faculdade de Medicina de Botucatu, da Unesp, que receberam indicação de oxigenoterapia e internação na enfermaria de Pediatria, no período entre maio e setembro de 2005 , independentemente de sexo e idade. Foram excluídas as crianças em uso crônico de oxigênio e aquelas submetidos à ventilação mecânica.

A pesquisa foi aprovada pelo Comitê de Ética em Pesquisa da referida instituição e as crianças participantes do estudo foram incluídas somente após autorização por escrito dos pais ou responsáveis.

Dos 8.709 atendimentos realizados no período, foram selecionadas 2.769 pacientes com hipótese diagnóstica relacionada a doenças do trato respiratório superior e/ou inferior, com indicação de oxigenoterapia e internação na enfermaria de Pediatria.

O uso do oxigênio foi avaliado por meio de um protocolo elaborado para este fim, preenchido pelo pesquisador que coletou os dados clínicos anotados pela equipe médica no pronto-socorro e na enfermaria de Pediatria. Foram enfatizados os 
seguintes parâmetros: dados pessoais do paciente, diagnóstico à admissão (baseado no encontro de infecção de vias aéreas inferiores com ou sem insuficiência respiratória, estertores e/ou broncoespasmo e exames subsidiários), indicações clínicas e laboratoriais para o emprego da oxigenoterapia, métodos de administração e monitoração contínua da oxigenoterapia por oxímetro de pulso, fluxo de oxigênio requerido (L/min), evolução do paciente com o tratamento, duração da terapêutica e indicação para interrupção da oxigenoterapia.

Os critérios adotados no Serviço para o uso de oxigenoterapia foram saturação de oxigênio $<90 \%$ e frequência respiratória elevada para a idade.

Os resultados foram tabulados em planilhas Excel $^{\circledR} \mathrm{e}$ descritos em média, mediana e desvio padrão (dp).

\section{Resultados}

No período de maio a setembro de 2005 , foram realizados 8.709 atendimentos no pronto-socorro pediátrico do hospital, entre os quais foram identificados 2.769 (32\%) casos de doenças do trato respiratório superior e/ou inferior, sendo $53 \%$ crianças do sexo masculino. A maioria dos diagnósticos de doenças respiratórias foi de vias aéreas superiores (57\%), havendo 498 (18\%) crianças com comprometimento de vias aéreas superiores e inferiores e 699 (25\%) com diagnóstico de doenças de vias aéreas inferiores. Foram excluídos cinco pacientes por falha no registro do diagnóstico.

Das 2.769 fichas selecionadas para o estudo, 97 (4\%) pacientes tiveram indicação de internação na enfermaria de Pediatria e, destes, 62 (64\%) receberam oxigenoterapia. Cerca de 60\% das crianças que receberam oxigenoterapia eram do sexo masculino. A idade variou de 2 meses a 14 anos; a distribuição da idade por faixa etária de tais crianças está mostrada na Tabela 1.

Todos os pacientes que necessitaram de oxigenoterapia apresentavam doenças respiratórias baixas e as indicações de uso foram saturação de oxigênio em ar ambiente $<90 \%$ e frequiência respiratória (FR) acima da esperada para a faixa etária (média de 53 movimentos por minuto). Entre os sinais e sintomas clínicos destes pacientes, estavam presentes dispnéia em 25 (40\%), taquipnéia em 26 (42\%), tosse em $20(32 \%)$, cianose em cinco (8\%) e apnéia em três (5\%).

A principal causa da hipoxemia foi a pneumonia, confirmada pelo quadro clínico e radiológico em 52 (84\%) pacientes. Em 16 (31\%) casos, havia associação do quadro infeccioso com sibilância. Outras causas da hipoxemia foram: asma em cinco (8\%) casos, bronquiolite em quatro $(6 \%)$ e traqueomalácia em um $(2 \%)$.

As prescrições de oxigênio foram feitas por médicos em sua totalidade, que observavam os pacientes diariamente e os monitoravam por meio de oxímetro de pulso. Apenas em seis $(10 \%)$ casos, foram realizadas gasometrias para complementar o controle de saturação de oxigênio observado com o uso do oxímetro.

Entre os métodos de administração de oxigênio, observou-se que o cateter nasal foi usado em 58 (94\%) crianças, isoladamente ou em associação. A tenda de oxigênio ou a máscara foi utilizada inicialmente em dois (3\%) pacientes cada uma. $\mathrm{O}$ uso desses métodos não foi feito de forma contínua, permanecendo apenas durante o primeiro dia e substituídos por cateter nasal até o fim da oxigenoterapia (Tabela 2). O tempo mediano de uso do oxigênio foi seis

Tabela 1 - Distribuição das crianças internadas em uso de oxigênio segundo faixa etária em meses

\begin{tabular}{lrr}
\hline Faixa etária & $\mathbf{N}$ & $\%$ \\
\hline $0-\mid 3$ & 13 & 21 \\
$3-\mid 6$ & 13 & 21 \\
$6-\mid 12$ & 18 & 29 \\
$12-\mid 24$ & 3 & 5 \\
$24-\mid 36$ & 5 & 8 \\
$36-\mid 48$ & 3 & 5 \\
$>48$ & 7 & 11 \\
Total & $\mathbf{6 2}$ & $\mathbf{1 0 0}$ \\
\hline
\end{tabular}

Tabela 2 - Distribuição das formas de administração de oxigênio segundo faixa etária em meses

\begin{tabular}{lcccc}
\hline Forma de administração $\mathbf{O}_{\mathbf{2}}$ & $\mathbf{N}$ & Faixa etária & Idade (mediana) & \% \\
\hline Cateter nasal & 40 & $01-\mid 168$ & 8 & 65 \\
Tenda & 2 & $03-\mid 6$ & 4 & 3 \\
Máscara & 2 & $12-\mid 24$ & 18 & 3 \\
Cateter+tenda & 4 & $02-\mid 8$ & 4 & 6 \\
Cateter+máscara & 14 & $01-\mid 96$ & 20 & 23 \\
Total & $\mathbf{6 2}$ & $\mathbf{0 1 - | 1 6 8}$ & $\mathbf{8}$ & $\mathbf{1 0 0}$ \\
\hline
\end{tabular}


dias (variação: 1-28 dias). Os fluxos de oxigênio mais utilizados foram de $1 \mathrm{~L} / \mathrm{min}$ e $2 \mathrm{~L} / \mathrm{min}$. Em poucos casos houve variação de fluxo, com recomendação de $0,5 \mathrm{~L} / \mathrm{min}$ ou, até mesmo, taxas mais elevadas, de 3 a $5 \mathrm{~L} / \mathrm{min}$. No entanto, esses valores eram mantidos por curto período e, em seguida, o fluxo utilizado foi de 1 a $2 \mathrm{~L} / \mathrm{min}$.

A oxigenoterapia era suspensa quando a saturação de oxigênio em ar ambiente alcançava valores maiores que $90 \%$. A evolução de todos os pacientes internados na enfermaria e submetidos ao uso de oxigênio foi satisfatória, resultando em alta hospitalar, com média de 12 dias de internação.

\section{Discussão}

A estimativa mundial de pneumonia em crianças menores de cinco anos é ao redor de 150,7 milhões de casos novos por ano. Mais de $95 \%$ dos episódios ocorrem em países em desenvolvimento e aproximadamente 11 a 20 milhões (7 a 13\%) necessitam de internação hospitalar devido à gravidade ${ }^{(15)}$.

Os vírus respiratórios são reconhecidos como os agentes mais freqüentes nas infecções do trato respiratório inferior nos lactentes e são os principais determinantes de gravidade e hipoxemia. Estudos como o de Vieira et a ${ }^{(15)}$,em São Paulo, no hospital universitário, e o de Cintra $e t$ al ${ }^{(16)}$, em Ribeirão Preto, São Paulo, mostraram que o vírus sincicial respiratório (VSR) é o agente mais freqüente nos lactentes menores de 12 meses. O estudo de D'Elia et al ${ }^{(17)}$ também comprovou a participação do VSR nas infecções do trato respiratório inferior em lactentes, levando à sibilância e à necessidade de hospitalização e de oxigenoterapia.

No presente estudo, realizado entre maio e setembro de 2005, época relatada como a de maior prevalência do VSR, o diagnóstico de bronquiolite ocorreu somente em $7 \%$ de casos. Observou-se que, das 52 crianças que receberam oxigenoterapia por pneumonia, $71 \%$ eram lactentes até 12 meses e notou-se sibilos associados à pneumonia em $31 \%$, fatos que sugerem a natureza viral do processo pneumônico ${ }^{(16-18)}$. As infecções bacterianas do trato respiratório inferior raramente causam sibilância, apesar de que o contrário já foi observado em países em desenvolvimento ${ }^{(19)}$.

Os critérios utilizados para a hospitalização foram faixa etária, FR elevada para a idade e gravidade da pneumonia, marcada principalmente pela hipoxemia, como sugerido por consensos nacionais e internacionais ${ }^{(20-23)}$.

No presente estudo, optou-se por definir hipoxemia como valor de saturação de oxigênio $<90 \%$, critério este padronizado em nossa instituição e preconizado por algumas publicações ${ }^{(13,24,25)}$.
Em todos os casos de oxigenoterapia, as prescrições foram realizadas exclusivamente pela equipe médica, que se baseou em critérios como saturação de oxigênio $<90 \%$ em ar ambiente e FR elevada para a idade (a saber, FR $\geq 60 \mathrm{mov} / \mathrm{min}$ em $<2$ meses, $\geq 50 \mathrm{mov} / \mathrm{min}$ de 2 meses a 1 ano e $\geq 40 \mathrm{mov} / \mathrm{min}$ de 1-4 anos), indicações presentes nas recomendações amplamente conhecidas para o uso de oxigênio ${ }^{(21-23,26,27)}$. Além destas indicações, sinais clínicos como dispnéia, taquipnéia e tosse estavam presentes em muitos casos - informação em conformidade com trabalhos que sugerem a valorização destas queixas como indicativas de pneumonia em crianças menores de 5 anos $^{(20,22)}$. Em poucos casos, ocorreram sinais de maior gravidade como cianose e apnéia.

A equipe médica observava diariamente a evolução da criança para avaliar a resposta ao tratamento e identificar possíveis problemas como a posição incorreta da interface para administrar o oxigênio, a diminuição da oferta ou fluxo incorreto de oxigênio e a obstrução das vias aéreas por muco $^{(21,23)}$. Uma vez que a maior limitação do uso do oxigênio é sua toxicidade, dependente de fatores como pressão absoluta oferecida, duração da exposição e sensibilidade individual $^{(4,13,14)}$, a avaliação realizada diariamente pelos médicos demonstra conhecimentos destes efeitos colaterais.

Em estudo prévio na clínica de adultos desta instituição, observou-se que a oxigenoterapia era realizada de forma inapropriada quanto às indicações, monitoração e suspensão da terapia $^{(28)}$. Assim, a postura atual da equipe médica em seguir as recomendações preconizadas nacional e internacionalmente demonstra a melhora dos conhecimentos médicos.

Todas as crianças estudadas foram monitoradas com oxímetro de pulso. Esse método simples e não invasivo monitora de forma contínua a saturação arterial de oxigênio e permite diagnosticar a hipóxia, já que guarda estreita relação com a pressão parcial de oxigênio no sangue arterial ${ }^{(22,29)}$. Essa propriedade é de grande importância, já que, em muitos casos, níveis discretos de hipóxia podem não se acompanhar de elevação na FR, o que dificulta o reconhecimento de possível dificuldade respiratória na criança ${ }^{(13,28-31)}$. Dessa forma, o uso do oxímetro de pulso deve ser estimulado, garantindo a administração racional de oxigênio ${ }^{(22)}$.

O tempo mediano de 6 dias do uso do oxigênio foi semelhante ao encontrado na literatura ${ }^{(31)}$. A suspensão da terapia foi feita em todos os pacientes quando estes passavam a apresentar saturação de oxigênio em ar ambiente $>90 \%$, critério defendido em várias publicações ${ }^{(13,25)}$.

Pelo fato de ser um método seguro, simples, efetivo e de baixo custo, a utilização de cateteres nasais é recomendada 
por diversos autores ${ }^{(6,31)}$. O fato de os cateteres poderem ser desinfectados e esterilizados possibilita sua reutilização ${ }^{(31)}$. Além disso, os cateteres nasais empregados no estudo mostraram-se eficientes, mesmo utilizando fluxos menores de oxigênio. Tal característica é de extrema importância em países onde os recursos para a saúde são escassos, pois possibilita que a oxigenoterapia seja colocada em prática a custos menores, como já relatados em diversos trabalhos ${ }^{(6,31)}$. O Departamento de Materiais do Hospital das Clínicas da Faculdade de Medicina de Botucatu (Unesp) averiguou o custo despendido para o uso de oxigênio: $\mathrm{R} \$ 0,55 / \mathrm{m}^{3}$ de $\mathrm{O}_{2}$. Adequando esta informação ao tempo de uso de oxigênio por parte dos pacientes aqui estudados (mediana: 6 dias) e aos fluxos mais usados, concluiu-se que o custo do oxigênio utilizado para cada criança variou entre $\mathrm{R} \$ 4,75$ (US $\$ 2,48$ ) para $1 \mathrm{~L} / \mathrm{min}$ a $\mathrm{R} \$ 9,50(\mathrm{US} \$ 4,96)$ para $2 \mathrm{~L} / \mathrm{min}$.

A hipoxemia grave pode levar à morte e a seqüelas em qualquer idade e, freqüentemente, as infecções respiratórias graves cursam com hipoxemia. Nesse contexto, a valorização de dados clínicos, acrescidos de parâmetros objetivos, como a oximetria de pulso, permite o uso do oxigênio de forma racional, com custo baixo, ocasionando imenso benefício ao paciente pelo melhor prognóstico de sua doença. No presente estudo, observou-se que os critérios de indicação e administração de oxigenoterapia aqui descritos estão de acordo com os consensos atuais.

\section{Referências bibliográficas}

1. Saugstad OD. Is oxygen more toxic than currently believed? Pediatrics 2001;108:1203-5.

2. Usen $\mathrm{S}$, Webert M. Clinical signs of hypoxaemia in children with acute lower respiratory infection: indicators of oxygen therapy. Int J Tuberc Lung Dis 2001;5:505-10.

3. Muhe L, Webert M. Oxygen delivery to children with hypoxaemia in small hospitals in developing countries. Int J Tuberc Lung Dis 2001;5:527-32.

4. Hay WW Jr, Bell EF. Oxygen therapy, oxygen toxicity and the STOP-ROP trial. Pediatrics 2000;105:424-5

5. Lozano JM. Epidemiology of hypoxaemia in children with acute lower respiratory infection. Int J Tuberc Lung Dis 2001;5:496-504.

6. Frey B, Shann F. Oxygen administration in infants. Arch Dis Child Fetal Neonatal Ed 2003;88:F84-8.

7. Onyango FE, Steinhoff MC, Wafula EM, Wariua S, Musia J, Kitonyi J. Hypoxaemia in young Kenyan children with acute lower respiratory infection. BMJ 1993;306:612-5.

8. Brasil - Ministério da Saúde - DATASUS [homepage on the Internet]. Informações de Saúde - Indicadores de mortalidade. Mortalidade proporcional por infecção respiratória aguda em menores de 5 anos de idade em 2002 [cited 2007 Jul 14]. Available from: http://tabnet.datasus.gov.br/cgi/tabcgi. exe?idb2004/c07.def

9. II Consenso Brasileiro de Ventilação Mecânica. J Pneumol 2000;26 (Suppl 2): S1-2.

10. Troster EJ, Faria LS. Insuficiência respiratória aguda. In: Marcondes E, Vaz FA, Ramos JL, Okay Y, editores. Pediatria básica. $9^{\mathrm{a}}$ ed. São Paulo: Sarvier; 2003. Tomo II. p. 452-60.

11. E-medicine from WebMD [homepage on the Internet]. Neumeister M: Hyperbaric oxygen therapy [cited $2007 \mathrm{Jul}$ 14]. Available from: http://www.emedicine. com/plastic/topic526.htm

12. Bateman NT, Leach RM. Acute oxygen therapy. BMJ 1998;317:798-801.

13. Oliveira NF, Filho DAD. Tratamento da insuficiência respiratória aguda. In: Hirschheimer MR, Matsumoto T, Carvalho WB, editores. Terapia intensiva pediátrica. $2^{\mathrm{a}}$ ed. São Paulo: Atheneu; 1997. p. 285-91.

14. Rudan I, Tomaskovic L, Boschi-Pinto C, Campbell H; WHO Child Health Epidemiology Reference Group. Global estimate of the incidence of clinical pneumonia among children under five years of age. Bull World Health Organ 2004; 82:895-903.

15. Vieira SE, Stewien KE, Queiroz DA, Durigon EL, Török TJ, Anderson LJ et al. Clinical patterns and seasonal trends in respiratory syncytial virus hospitalizations in São Paulo, Brazil. Rev Inst Med Trop Sao Paulo 2001;43:125-31.

16. Cintra OA, Owa MA, Machado AA, Cervi MC, Figueiredo LT, Rocha GM et al. Occurrence and severity of infections caused by subgroup $A$ and $B$ respiratory syncytial virus in children in Southeast Brazil. J Med Virol 2001;65:408-12.
17. D'Elia C, Siqueira MM, Portes SA, Sant'Anna CC. Respiratory syncytial virus- associated lower respiratory tract infections in hospitalized infants. Rev Soc Bras Med Trop 2005;38:7-10.

18. Bronchodilators and other medications for the treatment of wheeze-associated illnesses in young children - WHO/ARI/ 93.29 [homepage on the Internet] Geneva: World Health Organization; 1993 [cited 2007 Jul 14]. Available from: http://www.who.int/child-adolescent-health/New_Publications/CHILD_HEALTH/ WHO_ARI_93.29.htm

19. Brasil - Ministério da Saúde. Manual de normas de assistência e controle das infecções respiratórias agudas. $4^{a}$ ed. Brasília (DF): Ministério da Saúde; 1994.

20. British Thoracic Society Standards of Care Committee. British Thoracic Society guidelines for the management of community acquired pneumonia in childhood. Thorax 2002;57 (Suppl 1):i1-24.

21. Sociedade Brasileira de Pneumologia. Diretrizes brasileiras em pneumonia adquirida na comunidade em pediatria. J Bras Pneumol 2007;33 (Suppl 1):S31-50.

22. WHO. Pocket book of hospital care for children: guidelines for management of common illnesses with limited resources. Geneve: World Health Organization 2005

23. Piva JP, Garcia PC, Santana JC, Barreto SS. Respiratory failure in the child. J Pediatr (Rio J) 1998;74 (Suppl 1): S99-112.

24. American Academy of Pediatrics Subcommittee on Diagnosis and Management of Bronchiolitis. Diagnosis and management of bronchiolitis. Pediatrics 2006;118:1774-93.

25. Fulmer JD, Snider GL. American College of Chest Physicians/National Heart, Lung, and Blood Institute national conference on oxygen therapy. Heart Lung 1984;13:550-62.

26. Brasil - Ministério da Saúde. Tratamento de pneumonias em hospitais de pequeno e médio portes. Brasília: Ministério da Saúde; 1996.

27. Cogni AL, Ponce D, Godoy I. Oxygen therapy in a university hospital in Brazil. Am J Med 2000;108:598-9.

28. Mower WR, Sachs C, Nicklin EL, Baraff LJ. Pulse oximetry as a fifth pediatric vital sign. Pediatrics 1997;99:681-6.

29. Rees PJ, Dudley F. Oxygen therapy in chronic lung disease. BMJ 1998;317:871-4.

30. Weber MW, Palmer A, Oparaugo A, Mulholland EK. Comparison of nasa prongs and nasopharyngeal catheter for the delivery of oxygen in children with hypoxemia because of a lower respiratory tract infection. J Pediatr 1995; 127:378-83.

31. Qazi S. Oxygen therapy for acute respiratory infections in young children. Indian Pediatr 2002;39:909-13. 